\title{
A novel rare copy number variant of the $A B C F 1$ gene identified among dengue fever patients from Peninsular Malaysia
}

B.P. Hoh', S.S. Sam², S.H. Umi ${ }^{1}$, M. Mahiran ${ }^{3}$, N.Y. Nik Khairudin ${ }^{4}$, S. Rafidah Hanim ${ }^{5}$ and S. AbuBakar ${ }^{2,6}$

${ }^{1}$ Institute of Medical Molecular Biotechnology, Faculty of Medicine, Universiti Teknologi MARA, Sungai Buloh Campus, Jalan Hospital, Sungai Buloh, Selangor, Malaysia

${ }^{2}$ Department of Medical Microbiology, Faculty of Medicine, University of Malaya, Kuala Lumpur, Malaysia

${ }^{3}$ Department of Medicine, Hospital Kota Bharu, Kelantan, Malaysia

${ }^{4}$ Department of Paediatrics, Hospital Kota Bharu, Kelantan, Malaysia

${ }^{5}$ Department of Microbiology and Parasitology, School of Medical Science, Health Campus, Universiti Sains Malaysia, Kota Bharu, Kelantan, Malaysia

${ }^{6}$ Tropical Infectious Disease Research and Education Centre,

Faculty of Medicine, University of Malaya, Kuala Lumpur, Malaysia

Corresponding author: B.P. Hoh

E-mail: hbpeng@salam.uitm.edu.my

Genet. Mol. Res. 13 (1): 980-985 (2014)

Received December 18, 2012

Accepted July 6, 2013

Published February 19, 2014

DOI http://dx.doi.org/10.4238/2014.February.19.9

\begin{abstract}
Copy number variation (CNV) is a form of genetic variation in addition to single nucleotide polymorphisms. The significance of CNV in the manifestation of a number of diseases is only recently receiving considerable attention. We genotyped 163 dengue patients from Peninsular Malaysia for genes possibly linked to dengue infection using quantitative real-time PCR. Here, we report a serendipitous discovery of a novel rare CNV of the $A B C F 1$ gene among the dengue patients. Among these patients, two had a gain of
\end{abstract}


1 copy $(\mathrm{CN}=3)$ and one had lost 1 copy $(\mathrm{CN}=1)$, indicating that a rare $\mathrm{CNV}$ of the $A B C F 1$ gene was detected among dengue patients from Peninsular Malaysia. Although the gene is suspected to regulate inflammatory responses and pathogen-induced cytokine storm, its relevance to dengue requires further investigation.

Key words: Rare copy number variation; $\mathrm{ABCF} 1$; MHC; qPCR

\section{INTRODUCTION}

Copy number variation $(\mathrm{CNV})$ is an alternative form of genetic variation that, in addition to single-nucleotide polymorphisms, has recently attracted much interest in the genetic research community. CNV has been recognized for a long time at the cytogenetic level, but its impact at the genome-wide scale has not been fully appreciated until recently. CNV covers segments of DNA that contain insertions, deletions, duplications, and complex multisite variants, and it typically ranges from $0.5-1.0 \mathrm{~kb}$ to several megabases in size (Redon et al., 2006; Lee and Scherer, 2010). The copy number of protein-coding genes contributes significantly to phenotypic variability and can be strikingly different between individuals, with reports of 10-fold changes in copy number of several genes (Korbel et al., 2008), and such changes influence the mRNA and protein expression that affect gene dosage (Stranger et al., 2007). It has been suggested that CNV is enriched in "environmentally sensitive" genes such as those involved in the immune defense system. The major histocompatibility complex/human leukocyte antigen system (MHC/HLA) is one of the most complex and variable regions in the human genome. To date, information regarding CNV in this region is relatively scarce.

The human ATP-binding cassette (ABC) transporters are one of the largest family of transporter genes, and comprise 49 members belonging to 7 subfamilies designated as A-G (Wang et al., 2011). This gene family transports a wide range of substrate molecules across intra- and extra-cellular membranes (Dean and Allilmets, 2001) and serves as an important component of cellular defense mechanisms. Many of these genes have been attributed to a number of autoimmune and inflammatory diseases (Dean and Allilmets, 2001; Ota et al., 2007; Paladini et al., 2009); drug resistance (Saito et al., 2009); and a variety of diseases with complex or Mendelian genetics (Gillet et al., 2007). The ATP-binding cassette subfamily F member $1(A B C F 1)$ gene is a member of the GCN20 subfamily, located at 6p21.33 adjacent to HLA-E in the MHC region (Paladini et al., 2009), with a size of $\sim 21$ $\mathrm{kb}$ (Figure 1). This gene is not well studied, and, hence, knowledge on its functional role is relatively limited (Dean and Allilmets 2001; Ota et al., 2007; Wang et al., 2011). However, it is believed that the ABCF1 protein may be regulated by tumor necrosis factor-alpha and may play a role in the enhancement of protein synthesis and the inflammation process; thus, ABCF1 may have a role in several autoimmune diseases (Richard et al., 1998; Wang et al., 2011).

In an attempt to identify potential genes that predispose individuals to severe dengue infection, we serendipitously identified a novel rare $\mathrm{CNV}$ of the $A B C F 1$ gene. To date this CNV has not been previously reported elsewhere such as in the Database of Genomic Variants (DGV; http://projects.tcag.ca/variation/). 


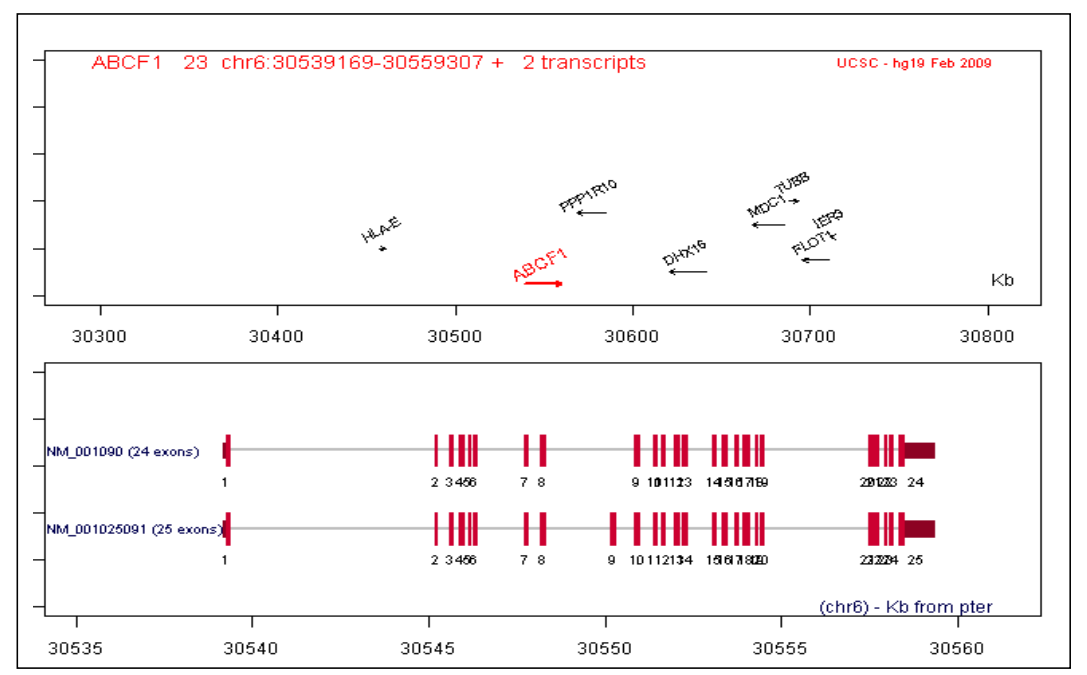

Figure 1. Location of ABCF1 from UCSC Genome Browser (http://genome.ucsc.edu/).

\section{MATERIAL AND METHODS}

In total, 163 hospitalized patients with laboratory-confirmed dengue infection (either dengue fever or dengue hemorrhagic fever) were enrolled in this study. These subjects were recruited from the Hospital Universiti Sains Malaysia (HUSM), Hospital Kota Bharu, and Universiti Malaya Medical Centre (UMMC) as study participants to investigate genetic susceptibility to dengue infection. The study was approved by the respective Research and Ethics Committee, i.e., University Malaya (UM) (reference No. 607.8), Universiti Sains Malaysia (USM) [USMKK/PPP/JePeM [211.3.(6)], and Ministry of Health (NMRR-09-1128-4211). All subjects provided informed and written consent to the genetic study. Three milliliters of peripheral blood was withdrawn from the recruited subjects and genomic DNA was extracted from either whole or clotted blood.

The copy number of the $A B C F 1$ gene in each patient was determined using relative quantitative real-time polymerase chain reaction (qRT-PCR). Amplification of the $A B C F 1$ gene and a reference gene, encoding telomerase reverse transcriptase (TERT), was performed simultaneously using a pre-designed TaqMan copy number assay (targeting a DNA region close to the central region of the gene, chr6:30656260; Figure 2) and TaqMan copy number reference assay (Applied Biosystems), respectively. The qRT-PCR mix, containing $4 \mu \mathrm{L}$ of each DNA sample (5 $\mathrm{ng} / \mu \mathrm{L}$ ), was prepared according to the manufacturer protocol and was run using the default thermal cycling conditions. All samples were measured in triplicates. The amplification efficiencies for the $A B C F 1$ and $T E R T$ genes were assessed by constructing a standard curve with a serial dilution of a reference DNA sample. After PCR amplification, data files containing the sample replicate $\mathrm{C}_{\mathrm{T}}$ values for each reporter dye were exported into the ABI Copy Number Caller software (Applied Biosystems) to determine the gene copy number in each sample. The software provides the statistical analysis by calculating the confidence values for each copy number call using the maximum likelihood algorithm. Samples were excluded from further analysis if they failed to give PCR products after 3 amplification attempts. 


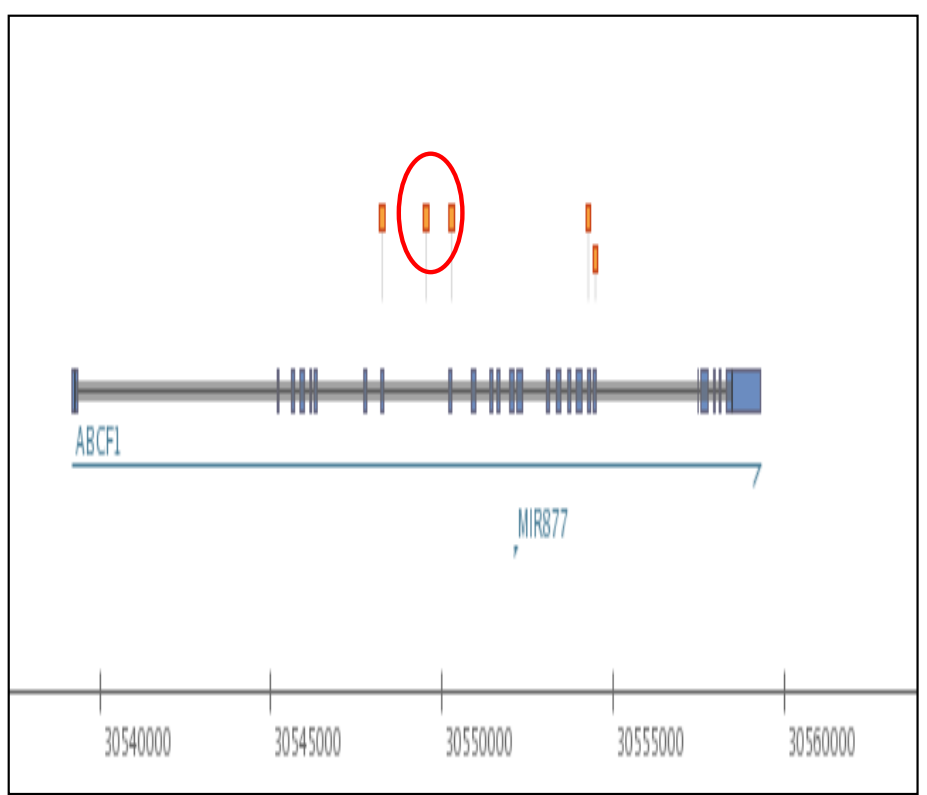

Figure 2. Location of $\mathrm{ABI} C \mathrm{CN}$ assay TaqMan Probe (http://www5.appliedbiosystems.com/tools/alignMap/ alignMap.rb?viewerAxisEntityType=CNV\&viewerAxisEntity=Hs02191244_cn\&gene_id=23).

\section{RESULTS AND DISCUSSION}

Of the 163 subjects genotyped, two were identified to have gained 1 copy of the $A B C F 1$ gene $(\mathrm{CN}=3)$, whereas loss of 1 copy was detected in one subject (Table 1$)$. Figure 3 shows the unrounded copy number calls and estimated CNV in the $A B C F 1$ gene.

Table 1. Copy numbers observed in the ABI TaqMan Copy Number Assay.
\begin{tabular}{lc}
\hline Samples I/D & CN ABI Copy Number Assay \\
\hline M5 & 3 \\
& $(2.81 ; 0.2)$ \\
M20 & 3 \\
D297 & $(2.85 ; 0.04)$ \\
& 1 \\
\end{tabular}

*Unrounded copy number values calculated using the relative quantification, standard deviation, are shown in parentheses.

Although CNVs in the regions of the MHC class II and class III genes have been recognized (Traherne, 2008), the CNV status in the class I region, where the $H L A-E$ and $A B C F I$ genes are located, remains to be identified. To date, no description of CNV in the $A B C F 1$ exists in the Database of Genomic Variants (DGV; http://projects.tcag.ca/variation/); therefore, the discovery of a $\mathrm{CNV}$ in the $A B C F 1$ gene among the dengue patient cohort was unexpected. $A B C F 1$ has previously been identified as an important gene in the regulation of inflammatory responses (Ota et al., 2007; Paladini et al., 2009) especially in pathogen-induced cytokine storm (Wilcox, 2010). These events are prominent features of severe dengue infection, but the 
A

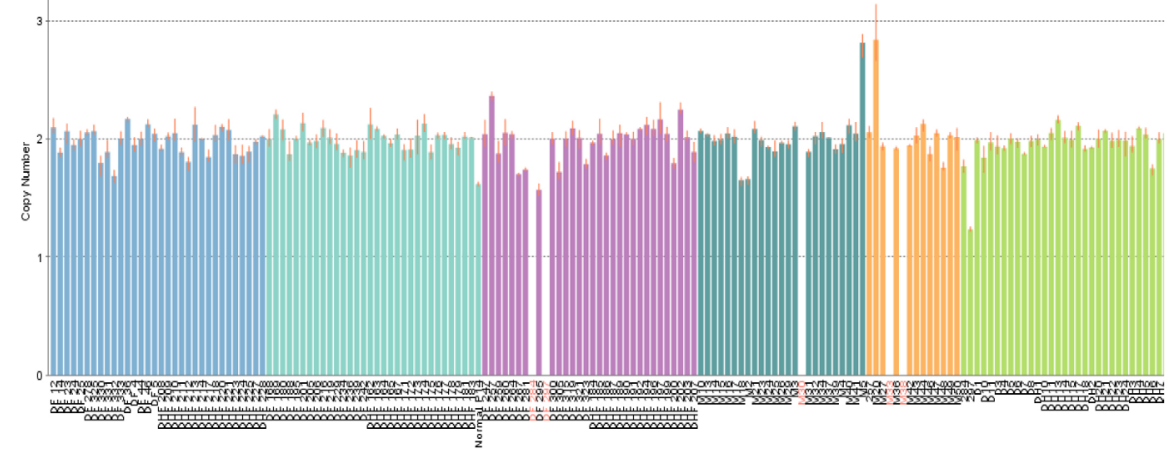

B

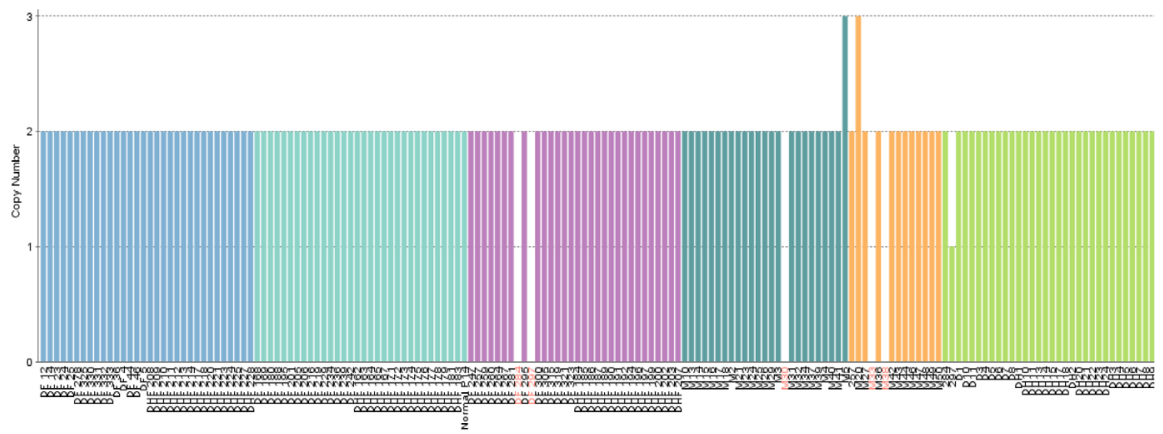

Figure 3. Copy number variation of the $A B C F 1$ gene obtained from ABI TaqMan qRT-PCR copy number assay. A. Unrounded copy number; B. predicted copy number by ABI Copy Number Caller. Samples highlighted in red failed in the PCR assays and were repeated for three attempts to confirm the presence of copy number calls (data not shown). We suspected that the failure of these samples was due to bad quality of DNA samples instead of $\mathrm{CN}=0$.

relevance of the $A B C F 1 \mathrm{CNV}$ to the outcome of severe dengue infection cannot be ascertained until a larger number of samples are analyzed.

In summary, we observed a novel rare $\mathrm{CNV}$ in the $A B C F 1$ gene in 3 subjects with dengue infection in Peninsular Malaysia. CNV in the $A B C F 1$ gene has not been previously reported. However, its relevance to dengue infection cannot be ascertained because of inadequate sample size.

\section{ACKNOWLEDGMENTS}

Research supported by the Fundamental Research Grant Scheme (FRGS) of the Ministry of Higher Education in Malaysia in 2007 (\#203/PPSP/6171107) and 2010 [\#600-RMI/ST/ FRGS 5/3/Fst (\#69/2009)]. We acknowledge the Ministry of Science, Technology, and Innovation of Malaysia for the Malaysia Genome Institute initiative grant \#07-05-MGI-GMB015 and the Ministry of Higher Education for Long-Range Grant Scheme (LRGS) 2011 (LRGS/ TD/2011/UM/Penyakit_Berjangkit). We thank all the subjects who volunteered for this study. We also would like to thank Professor Stephen W. Scherer and Dr. Christian Marshall from the Toronto Centre for Applied Genomics (TCAG) for their helpful advice and support. Finally, we thank the peer reviewers for their helpful comments and constructive suggestions. 


\section{REFERENCES}

Dean M and Allikmets R (2001). Complete characterization of the human ABC gene family. J. Bioenerg. Biomembr. 33: 475-479.

Gillet JP, Efferth T and Remacle J (2007). Chemotherapy-induced resistance by ATP-binding cassette transporter genes. Biochim. Biophys. Acta 1775: 237-262.

Korbel JO, Kim PM, Chen X, Urban AE, et al. (2008). The current excitement about copy-number variation: how it relates to gene duplications and protein families. Curr. Opin. Struct. Biol. 18: 366-374.

Lee C and Scherer SW (2010). The clinical context of copy number variation in the human genome. Expert Rev. Mol. Med. 12: e8.

Ota M, Katsuyama Y, Hamano H, Umemura T, et al. (2007). Two critical genes (HLA-DRB1 and ABCF1) in the HLA region are associated with the susceptibility to autoimmune pancreatitis. Immunogenetics 59: 45-52.

Paladini F, Belfiore F, Cocco E, Carcassi C, et al. (2009). HLA-E gene polymorphism associates with ankylosing spondylitis in Sardinia. Arthritis Res. Ther. 11: R171.

Redon R, Ishikawa S, Fitch KR, Feuk L, et al. (2006). Global variation in copy number in the human genome. Nature 444: 444-454.

Richard M, Drouin R and Beaulieu AD (1998). ABC50, a novel human ATP-binding cassette protein found in tumor necrosis factor-alpha-stimulated synoviocytes. Genomics 53: 137-145.

Saito A, Kawamoto M and Kamatani N (2009). Association study between single-nucleotide polymorphisms in 199 drug-related genes and commonly measured quantitative traits of 752 healthy Japanese subjects. J. Hum. Genet. 54: 317-323.

Stranger BE, Forrest MS, Dunning M, Ingle CE, et al. (2007). Relative impact of nucleotide and copy number variation on gene expression phenotypes. Science 315: 848-853.

Traherne JA (2008). Human MHC architecture and evolution: implications for disease association studies. Int. J. Immunogenet. 35: 179-192.

Wang LL, Liu YH, Meng LL, Li CG, et al. (2011). Phenotype prediction of non-synonymous single-nucleotide polymorphisms in human ATP-binding cassette transporter genes. Basic Clin. Pharmacol. Toxicol. 108: 94-114.

Wilcox SM (2010). The Function of ABCF1 in Immunity and Mouse Development. Doctoral thesis, University of British Columbia, Columbia. 